\title{
Aetiopathogenesis of liver changes in the course of cystic fibrosis, considering disturbances of the bile acid profile as well as genetic and immunological factors
}

\author{
Sabina Więcek, Halina Woś, Urszula Grzybowska-Chlebowczyk \\ Department of Paediatrics, Medical University of Silesia, Katowice, Poland
}

Prz Gastroenterol 2013; 8 (5): 290-294

DOI: $10.5114 /$ pg.2013.38730

Key words: liver changes, cystic fibrosis, children

Address for correspondence: Sabina Więcek MD, PhD, Department of Paediatrics, Medical University of Silesia, 16 Medyków St, 40-752 Katowice, Poland, phone: +48 600168 165, fax: +48 32207 17 21, e-mail: sabinawk@wp.pl

\begin{abstract}
Liver changes observed in the course of cystic fibrosis comprise a group of complex processes of fibrosis, inflammation, remodelling, apoptosis and cholestasis as a result of abnormal functioning of the cystic fibrosis transmembrane conductance regulator (CFTR) protein, immunological reactions and response to oxidation stress. Liver lesions are only observed in 5-20\% of patients with diagnosed cystic fibrosis; however, they increase mortality, reduce the lifespan and deteriorate the quality of life. Liver diseases are the most common extrapulmonary causes of death in patients with cystic fibrosis. The aim of the study was to analyse the hitherto performed studies on the aetiopathogenesis of liver changes in the course of cystic fibrosis considering disturbances of the bile acid profile as well as genetic and immunological factors.
\end{abstract}

\section{Introduction}

Liver changes observed in the course of cystic fibrosis (CF) comprise a group of complex processes of fibrosis, inflammation, remodelling, apoptosis and cholestasis as a result of abnormal functioning of the CFTR protein, immunological reactions and response to oxidative stress. So far, the complexity of processes occurring in the liver and bile ducts in the course of cystic fibrosis has not been elucidated [1, 2].

Liver changes associated with cystic fibrosis usually occur in the first decade of life. Hepatic cirrhosis is diagnosed in approx. $10 \%$ of children ( $<18$ years of age) with cystic fibrosis, but in only $2 \%$ of adults suffering from this disease. The average age of cirrhosis manifestation is approx. 10 years [3].

Liver lesions are only observed in $5-20 \%$ of patients with diagnosed cystic fibrosis; however, they increase mortality, reduce the lifespan and deteriorate the quality of life.

Liver diseases are the most common extrapulmonary causes of death in patients with cystic fibrosis.
However, in as many as $72 \%$ of patients suffering from cystic fibrosis the autopsy reveals focal liver fibrosis. Lamireau observed liver changes in $18 \%$ of patients after 2 years, in $29 \%$ after 5 years and in $41 \%$ after 12 years following the diagnosis of CF [3].

\section{Pathophysiological changes of bile acids in the course of cystic fibrosis}

Impaired expression of CFTR protein on the top cholangiocyte membranes, within the bile duct epithelium and gallbladder epithelium, results in changes of bile composition (water content and electrolyte disturbances, bile pH changes), abnormal bile transport and retention of toxic bile acids (taurocholic acid). This induces chemokines responsible for inflammation and fibrosis. Monocyte chemotactic protein in hepatocytes and cholangiocytes stimulates the process of stellate cells chemotaxis, leading to peribiliary fibrogenesis $[4,5]$.

Changing the bile acid profiles into hydrophobic ones is connected with stimulation of the enterohepatic circulation. Biliary secretion of bilirubin monoglucuronides, bile salts, phospholipids and cholesterol is 
considerably higher in patients with cystic fibrosis. Bile acid salts that become deposited within cholangiocytes and bile ducts are mostly iron and calcium glucorates. Interestingly, iron and calcium glucurates precipitate at unfavourable bile $\mathrm{pH}$. Hyperbilirubinobilia is a risk factor for increased intrahepatic hydrolysis due to endogenous glucuronidase and deposits in cholangiocytes and bile ducts. Probably increased loss of bile salts in faeces also leads to formation of more hydrophobic bile salts in bile ducts and to hyperbilirubinobilia. An important role is also played by disturbed bile salt/phospholipid ratio and endogenous hydrolysis of glucuronates. Insoluble salts form a black pigment included in bile stones. No connection has been demonstrated between disturbed cholesterol metabolism and abnormal bile composition in a murine model of cystic fibrosis [6].

In a healthy liver, toxicity of hydrophobic bile acids is regulated by formation of micelles containing bilirubin, cholesterol and phospholipid proteins as well as by bile hydration, alkalization, the presence of mucins and normal bile outflow from the liver. It has been demonstrated that patients with hepatic lesions in the course of cystic fibrosis (cystic fibrosis liver diseases - CFLD) have lower levels of ursodeoxycholic acid in favour of taurine conjugates of cholic acid. Perhaps, an additional role is played by fibroblast growth factor 19 , which is produced by enterocytes in the terminal ileum.

Toxic effects of bile acids may cause apoptosis of hepatocytes, which results in activation of extracellular matrix cells and transformation into myofibroblasts. These processes lead to activation of caspases (proteases and endonucleases) and degradation of dead hepatocytes which are engulfed by Kupffer cells and macrophages. Kupffer cells produce TNF- $\alpha$ which activates myofibroblasts participating in the process of fibrosis $[6,7]$.

Hepatic fibrosis is a result of hemodynamic imbalance between synthesis (fibrogenesis) and degradation (fibrolysis) of the connective tissue components, leading to excessive and modified accumulation of extracellular matrix elements (ECM) in this organ. Hepatic stellate cells are the main source of fibrotic tissues - type I, III and IV collagen, and glycoproteins (laminin, elastin, hyaluronates, vesicant). An additional role in remodelling is played by the production of collagenases, metalloproteinases and their inhibitors as well as plasminogen activator inhibitor type 1 (PAI-1). The question why this balance becomes disturbed in patients with cystic fibrosis has not been fully answered yet. Under normal conditions repair processes are initiated. Concurrent inflammatory processes involving intercellular adhesion molecule 1 (ICAM1), lymphocytes, macrophages and cytokines are also involved. Increased levels of T lymphocytes CD4, CD3, CD8, NK CD56 and macrophages
CD68 have been observed in intrahepatic cholestases. It is also known that interleukins 4, 10 and 18 (IL-4, IL-10 and IL-18) levels are elevated in these diseases [6-8].

Portal hypertension is a result of disturbed balance between vasodilators ( $\mathrm{NO}, \mathrm{CO}, \mathrm{H}_{2} \mathrm{~S}$, prostaglandins) and vasoconstrictors (endothelin 1).

It is also possible that some role is played by intestinal endocrine factor 19, participating in the regulation of bile acid synthesis, and monocyte chemotactic protein (MCP-1) induced by taurocholic acid and contributing to the process of fibrogenesis.

Additional factors promoting the process of bile acid precipitation in the bile ducts are: decreased synthesis of bile acid salts, decreased absorption of bile acids from the small intestine, bile duct strictures hindering bile flow from the liver to the duodenum.

Orally administered ursodeoxycholic acid has a cytoprotective effect on cholangiocyte membranes and stimulates secretion of chlorine ions through calcium-activated chloride channels. It is a hydrophilic acid; it reduces the proportion of cholic acid in bile (below $5 \%$ ), decreases its synthesis and total circulating pool, and stimulates cholangiocytes and hepatocytes to secretion. Additionally, it exerts anti-apoptotic effects and reduces toxic effects of hydrophobic bile acids. It has been demonstrated that ursodeoxycholic acid administered at a dose of $20 \mathrm{mg} / \mathrm{kg}$ bw/day to patients with cystic fibrosis and coexisting focal biliary cirrhosis inhibits disease progression.

\section{Genetic factors}

The molecular background of hepatic and biliary changes in the course of cystic fibrosis has not been sufficiently elucidated.

\section{Type of CFTR gene mutation}

So far, no specific mutations related exclusively to liver damage in the course of cystic fibrosis have been detected. Such mutations are usually "serious mutations" of the CFTR gene (delta F508, G524X, N1303K, CFTRdel21kB, 1811+1G-> C) $[9,10]$. Mutation delta 508 is a special risk factor of hepatic disease development in patients with cystic fibrosis; it stimulates increased loss of bile acids in faeces and enhances formation of more hydrophobic bile salts. Low $\mathrm{pH}$ of the gallbladder bile and increased concentration of calcium bilirubinates - especially frequent in patients with this mutation - lead to high bile saturation and accumulation of the black colour in the gallbladder. On the other hand, coexistence of hepatic lesions and cystic fibrosis has not been described in any patients with $3849+10 \mathrm{kB}$ C->T mutation [11]. However, the clinical course in patients with diagnosed cystic fibrosis and the same mutation of the CFTR gene can 
be different. There is no strict correlation between phenotypes and genotypes. Other coexisting mutations and environmental factors probably have some impact.

\section{SERPINA1 gene}

The SERPINA1 gene is present in approx. $2 \%$ of patients with cystic fibrosis and in approx. $5 \%$ of patients with cystic fibrosis and coexisting hepatic lesions. The SERPINA1 gene is responsible for synthesis of serine protease inhibitor. Protein related to the $Z$ allele is accumulated within hepatocyte endoplasmic reticulum, leading to hepatocyte damage, inflammation and cirrhosis. In approx. $10 \%$ of $Z$ allele homozygotes, the accumulation of SERPINA gene protein results in neonatal hepatitis, and in $2-3 \%$ of cases in fibrosis and cirrhosis. A high risk of portal hypertension development has been demonstrated in patients having the $Z$ allele of the SERPINA 1 gene. Moreover, its presence is related to a risk of emphysema and chronic obstructive pulmonary disease. However, the lung function in patients with cystic fibrosis is not dependent on presence of the Z allele [4, 12-14].

\section{Plasminogen activator inhibitor 1 gene}

Plasminogen activator inhibitor belongs to a family of serine protease inhibitors. Its function is based on neutralization of active particles of tissue plasminogen activator and urokinase plasminogen activator by forming stable, inactive complexes. Plasminogen activator inhibitor 1 is mainly synthesized by endothelial cells, hepatocytes, megakaryocytes and smooth muscle cells in blood vessels. It has also some influence on fibrinolysis (conversion of inactive plasminogen protein into plasmin, an active protease that decomposes and removes fibrin deposits). Decreased PAI-1 levels were observed during viral hepatitis, fatty liver and alcoholic hepatitis, and primary biliary cirrhosis. On the other hand, decreased PAI-1 activity in cholestatic liver damage has been observed in murine models. Plasminogen activator inhibitor 1 is an acute-phase reactant. Its concentration correlates with the process of liver fibrosis. Mice with cholestasis induced by bile duct destruction have been examined. Plasminogen activator inhibitor 1 correlated with the levels of matrix metalloproteinases and plays an import ant role as a fibrosis process mediator in the course of cholestasis [1, 15-17].

\section{Genes related to metalloproteinases 1}

Metalloproteinases are members of a large family of multiple domain zinc endopeptidases. They belong to principal proteolytic enzymes digesting extracellular matrix components and numerous molecules on the cell surface, participating in physiological (apoptosis, angiogenesis) and pathological (fibrogenesis and fibrolysis) processes. Decreased levels of metalloproteinase inhibitor (TIMP1) were observed in children with biliary atresia. Decreased activity of metalloproteinases is observed in early phases of liver fibrosis, whereas during advanced processes of fibrosis increased values are detected (role of remodelling?). The levels of metalloproteinases, however, are influence by age, the growth process, renal clearance and bone metabolism $[1,8,18,19]$.

\section{Glutathione S-transferase P1 gene}

It has antioxidant activity. If a mutation of this gene is present, impaired detoxification capabilities are observed. Glutathione transferase plays a key role for the protection of hepatocytes against oxidation stress which influences the manifestation of hepatic lesions in patients with cystic fibrosis. The prevalence of mutations of the glutathione S-transferase P1 (GSTP1) gene is statistically higher in patients with cystic fibrosis and coexisting liver disease $[12,13]$.

\section{Mannose-binding lectin 2 (MBL2) gene}

Upon the mutation of this gene, increased liver exposure to toxic agents in patients with cystic fibrosis has been demonstrated $[13,20]$.

\section{Transforming growth factor $\beta$ gene - TGF- $\beta 1$ gene}

It has been demonstrated that TGF- $\beta$ is related to a high risk of liver fibrosis in the course of autoimmune hepatitis; however, there are no such observations for patients with cystic fibrosis $[4,18,21,22]$.

\section{UGT1A1 gene - uridyltransferase gene}

This gene, characteristic for Gilbert's syndrome, promotes development of lithiasis in patients with coexisting cystic fibrosis and Gilbert's syndrome [19].

Immunological factors - stellate cells are stimulated to produce collagen and proinflammatory cytokines.

\section{Role of chemokines}

Chemokines influence the activation of stellate cells both in vivo and in vitro. Factors stimulating proliferation are: monocyte chemotactic protein, tumor necrosis factor $\alpha$ (TNF- $\alpha$ ), platelet-derived growth factor, IL-1, and insulin-like growth factor. Fibrinogenic factors include: TGF- $\beta$, IL- 6 , and metalloproteinases. The sources of cytokines are: liver macrophages, endothelium cells, biliary duct epithelial cells, lymphocytes, blood platelets, hepatocytes and activated stellate cells [22, 23]. 


\section{Monocyte chemotactic protein (MCP1)}

It stimulates stellate cells to chemotaxis, and secondarily, activates fibrogenesis. Monocyte chemotactic protein MCP-1 levels correlate with the level of hydrophobic bile acids (taurocholic acid) in blood serum and hepatocytes. This protein increases at the early stages of fibrosis in the course of cystic fibrosis [4, 23].

\section{Macrophage inflammatory protein $1 \beta$ (MIP1B)}

It increases at the early stages of liver fibrosis (especially F1-F2 in liver biopsies) in patients with cystic fibrosis.

\section{Tumor growth factor $\beta$}

It plays both direct and indirect roles as a fibrogenic mediator participating in liver remodelling. Hepatic stellate cells are induced by TGF- $\beta$ to transform into myofibroblasts and to produce extracellular matrix. Tumor growth factor $\beta$ is one of the most important profibrogenic factors in many organs, including in the liver. It is activated by proteases and integrins. It influences the activity of metalloproteinases and plasminogen activator inhibitor 1 . Its activity correlates with the process of liver fibrosis $[18,21]$.

\section{Tumor necrosis factor $\alpha$}

Due to its influence on receptors, it activates the arachidonic acid cascade and increases the intracellular concentration of free radicals, leading to cellular apoptosis (including hepatocytes).

\section{Platelet-derived growth factor}

It belongs to peptide growth factors. Platelet-derived growth factor (PDGF) expression is observed on many mesenchymal cells; it is also a mitogenic and chemotactic agent for neutrophils, monocytes, smooth muscle cells, fibroblasts and mesangial cells. There is a close relationship between the level of PDGF expression and the stage of liver fibrosis. It is believed that PDGF and its receptors potentiate liver fibrosis via activation, proliferation and differentiation of satellite cells. It has been demonstrated that PDGF is a mediator of transformation of peribiliary cells into myofibroblasts, which contributes to the development of biliary cirrhosis in patients with cystic fibrosis [22, 23].

\section{Interleukins: IL-1, IL-6, IL-10}

They play a potential role in retransformation of myofibroblasts into hepatic stellate cells [22, 23].

\section{Summary}

Aetiopathogenesis of hepatic lesions in the course of cystic fibrosis is very complex and still unclear. The contributions of numerous genetic and immunological factors and changed bile acid profiles are observed. It is necessary to continue studies aimed at explaining the causes of hepatic lesions in the course of cystic fibrosis, which will reduce mortality, prolong the life span and improve the quality of life in such patients.

\section{References}

1. Rudnick D. Cystic fibrosis - associated liver disease: when will the future be now? J Pediatr Gastroenterol Nutr 2012; 3: 312-7.

2. Rowland M, Gallagher C, O'Laoide R, et al. Outcome in cystic fibrosis liver disease. Am J Gastroenterol 2011; 106: 104-9.

3. Lamireau T, Monnerau S, Martin S, et al. Epidemiology of liver disease in cystic fibrosis: a longitudinal study. J Hepatol 2004; 41: 920-5.

4. Pereira T, Lewindon P, Greer R, et al. Transcriptional basis for hepatic fibrosis in cystic fibrosis-associated liver disease. J Pediatr Gastroenterol Nutr 2012; 3: 328-35.

5. Colombo C, Russo M, Zazzeron L, Romano G. Liver disease in cystic fibrosis. J Pediatr Gastroenterol Nutr 2006; 43: 49-55.

6. Freudenberg F, Broderick A, Yu B, et al. Pathophysiological basis of liver disease in cystic fibrosis employing a Delta F508 mouse model. Am J Gastrointest Liver Physiol 2008; 294: 1411-20.

7. Tanner M, Taylor C. Liver disease in cystic fibrosis. Arch Dis Child 1995; 72: 281-4.

8. Pereira T, Walsh M, Lewindon P, et al. Paediatric cholestatic liver disease: diagnosis, assessment of disease progression and mechanisms of fibrogenesis. World J Gastrointest Pathophysiol 2010; 15: 69-84.

9. Arkwright P, Pravica V, Geraghty P, et al. End-organ dysfunction in cystic fibrosis. Am J Respir Crit Care Med 2003; 167: 384-9.

10. Fustik S, Traikovska M, Jakovska T, et al. Screening for liver disease in cystic fibrosis: analysis of clinical and genetic risk factors for its development. Turk J Pediatr 2008; 50: 526-32.

11. Wilschanski M, Rivlin J, Cohen S, et al. Clinical and genetic risk factors for cystic fibrosis-related liver disease. Pediatrics 1999; 103: 52-7.

12. Herrmann U, Dockter G, Lammert F. Cystic fibrosis-associated liver disease. Best Practise Res Clin Gastroenterol 2010; 24: 585-92.

13. Bartlett J, Friedmann K, Ling S, et al. Genetic modifiers of liver disease in cystic fibrosis. JAMA 2009; 9: 1076-83.

14. De Haan W. A marker associated with increased risk for severe liver disease in cystic fibrosis. Clin Genet 2010; 77: 434-7.

15. Fitch P, Bennett B, Booth A, et al. Distribution of plasminogen activator inhibitor in normal liver, cirrhotic liver, and liver with metastases. J Clin Pathol 1994; 47: 218-21.

16. Canbay A, Feldstein A, Baskin-Bey E, et al. The caspase inhibitor IDN- attenuates hepatic injury and fibrosis in the bile duct ligated mouse. J Pharmacol Exper Ther 2004; 3: 1191-6.

17. Bergheim I, Guo L, Davis M, et al. Critical role of plasminogen activator inhibitor-1 in cholestasis liver injury and fibrosis. J Pharmacol Exp 2006; 316: 592-600. 
18. Raszeja-Wyszomirska J, Mieżyńska-Kurtycz J, Marlicz W, et al. Non invasive markers of non-alcoholic steatohepatitis. Pol Merk Lek 2008; 146: 166-9.

19. Lindblad A, Glaumann H, Strandvik B. Natural history of liver disease in cystic fibrosis. Hepatology 1999; 5: 1151-8.

20. Pereira T, Lewindon P, Smith J, et al. Serum markers of hepatic fibronegesis in cystic fibrosis liver disease. J Hepatol 2004; 41: 576-83.

21. Weng H, Ciuclan L, Liu Y, et al. Profibrogenetic transforming growth factor-beta/activin receptor like kinase 5 signaling via connective tissue growth factor expression in hepatocytes. Hepatology 2007; 46: 1257-70.

22. Tsukamoto $\mathrm{H}$. Cytokine regulation of hepatic stellate cells in liver fibrosis. Alcohol Clin Exp Res 1999; 23: 911-6.

23. Pinzani $M$, Marra F. Cytokine receptors and signaling in hepatic stellate cells. Semin Liver Dis 2001; 21: 397-416.

Received: 13.08 .2012

Accepted: 7.01.2013 